\title{
Capturing the Knudsen layer in continuum-fluid models of
}

\section{non-equilibrium gas flows}

Duncan A Lockerby

Department of Mechanical Engineering, King's College London, London WC2R 2LS, $U K$

Jason M Reese

Department of Mechanical Engineering, University of Strathclyde, Glasgow G1 1XJ, $U K$

Michael A Gallis

Engineering Sciences Center, Sandia National Laboratories, PO Box 5800, Albuquerque, NM 87185-0827, USA

\section{Introduction}

In hypersonic aerodynamics and microflow device design, the momentum and energy fluxes to solid surfaces are often of critical importance. However, these depend on the characteristics of the Knudsen layer — the region of local non-equilibrium existing up to one or two molecular mean free paths from the wall in any gas flow near a surface. While the Knudsen layer has been investigated extensively using kinetic theory ${ }^{1} 2^{3}$, the ability to capture it within a continuum-fluid formulation (in conjunction with slip boundary conditions) suitable for current computational fluid dynamics toolboxes would offer distinct and practical computational advantages.

The Navier-Stokes constitutive relations cannot, however, model the nonlinear stress/strain-rate behavior within the Knudsen layer. Although higher-order 
continuum equations, such as the Burnett equations, may provide an alternative, Kogan ${ }^{1}$ has shown that the Chapman-Enskog series (from which the Burnett and super-Burnett equations are derived) does not provide a solution to the Boltzmann equation in the Knudsen layer. Furthermore, higher-order continuum equations require additional boundary conditions to ensure a unique solution: without using results from other simulations or experiments, it is not a straightforward matter to choose these. Within continuum-fluid formulations for locally non-equilibrium gas flows, a phenomenological approach could therefore be a practical way of capturing the essential Knudsen layer structure. It is, after all, accepted and commonplace for phenomenological methods to be applied at wall boundaries (Maxwell's velocity slip and the classical non-slip boundary condition are partly phenomenological).

When using the Navier-Stokes equations to model a slightly rarefied gas flow, the most common approach is to account for (rather than model) the Knudsen layer by employing "fictitious" or "macro" slip boundary conditions at the wall $\left(u_{\text {slip }}^{*}\right.$, in Figure 1). If the "actual" or "micro" velocity slip $\left(u_{\text {slip }}\right)$ is applied at the wall, the prediction of the velocity both inside and outside the Knudsen layer is poor (the dash-dot line in Figure 1). Prescribing a fictitious velocity slip does, at least, provide an accurate solution outside the Knudsen layer (the dashed line in Figure 1). Kogan ${ }^{1}$, Cercignani ${ }^{2}$ and Sone ${ }^{3}$ all proposed such slip boundary conditions. (Sone intended his boundary condition to be used in conjunction with a tabulated Knudsen layer correction; this approach will discussed later.) 
The drawback of employing fictitious slip boundary conditions, however, is that some part of the flow field then necessarily becomes fictitious. For flows with higher global Knudsen numbers, the physical extent of the Knudsen layer increases and the fictitious element of a Navier-Stokes solution to the flow field therefore occupies a significant proportion of the entire flow. The error that this generates near the boundary is unacceptable because, in the majority of applications (from hypersonics and microfluidics), it is the accurate prediction of surface properties that is of paramount importance. Higher-order slip boundary conditions ${ }^{456}$ cannot remedy this problem because the error arises not from an inaccuracy in the slip model at the boundary, but from within the Navier-Stokes equations themselves (i.e. from the linearity of these constitutive relations).

Another current approach is to apply the Navier-Stokes equations with fictitious slip boundary conditions, as above, and then make a kinetic theory-based correction to either the resulting velocity field using pre-calculated values ${ }^{3}$ or to an averaged property of interest, such as mass flow rate ${ }^{4}$. In both approaches the Navier-Stokes calculation of the flow field is treated separately from the Knudsen layer correction; consequently, the presence of the Knudsen layer does not directly affect the flow solution. Furthermore, as these Navier-Stokes solutions require fictitious boundary conditions, some properties of the entire flow solution are likely to be partially in error even after the Knudsen layer corrections. 


\section{A wall-function approach}

We do not present here a physical model for the Knudsen layer, but rather a "wallfunction" technique, akin to that used in turbulent boundary-layer modeling, which can capture its essential features in computational fluid dynamics. We use a solution to the linearized Boltzmann equation for a wall-bounded shear flow of a monatomic gas as the basis for a new scaling law for the gas effective viscosity as a function of normal wall distance. While the Knudsen layer also affects the relationship between heat flux and temperature gradient, at this stage we restrict our attention to its effect on the stress/strain-rate relationship (noting that the variation of viscosity will also cause the thermal conductivity to vary).

For a planar wall (with diffuse molecular deflection) bounding a monatomic gas flow subject to a uniform shear stress, Cercignani showed ${ }^{2}$ that the linearized Boltzmann equation predicts a velocity profile, $u$, through the Knudsen layer of the form

$$
u=-\frac{\tau}{\mu}(x+\zeta-\lambda I(x / \lambda))
$$

where $x$ is the normal distance from the planar wall, $\tau$ is the uniform shear stress, $\mu$ is the viscosity, $\lambda$ is the mean free path, and $\zeta$ is a constant. For convenience, we propose a curve-fitted approximation to the velocity correction function, $I(x / \lambda)$ (evaluated by Cassell and Williams ${ }^{7}$ ) as follows:

$$
I(x / \lambda) \approx \frac{7}{20}\left(1+\frac{x}{\lambda}\right)^{-2}
$$


with a mean free path given by

$$
\lambda=\mu \sqrt{\frac{\pi}{2 p \rho}}
$$

where $\rho$ is the gas density and $p$ its pressure.

On differentiating Equation (1) a correction to the constitutive relations of the NavierStokes equations that is appropriate throughout the Knudsen layer can be obtained:

$$
\frac{\mathrm{d} u}{\mathrm{~d} x}=-\frac{\tau}{\mu} \Psi(x / \lambda)
$$

where the "wall-function", $\Psi(x / \lambda)$, is given by

$$
\Psi(x / \lambda)=1-\lambda \frac{\mathrm{d} I(x / \lambda)}{\mathrm{dx}} \approx 1+\frac{7}{10}\left(1+\frac{x}{\lambda}\right)^{-3}
$$

For a given shear stress, this wall-function generates a strain rate at the wall that departs some $70 \%$ from the Navier-Stokes predictions — decreasing to roughly $10 \%$ difference a distance of one mean free path into the gas. A consequence of this scaling of the gas viscosity is that the normal strain is affected similarly to the shear strain.

Since this wall-function is based on Cercignani's linearized Boltzmann solution for a uniform shear stress over a planar surface, strictly our model should be applicable only to low Mach number, low Knudsen number flows over planar surfaces with diffuse reflection at the solid boundaries. However, we can test the practicality of using this wall-function to improve predictions in more general flow configurations (see below). It should be noted that this is a preliminary investigation into developing straightforward techniques for incorporating Knudsen layer structure within 
conventional fluid dynamics simulations - a general wall-function approach cannot be expected to obtain predictions comparable with the accuracy of modern kinetic theory.

With the wall-function given by Equation (4), Maxwell's general slip condition ${ }^{8}$ should also be used:

$$
u_{s l i p}=-A_{1} \frac{2-\sigma}{\sigma} \lambda \frac{\tau}{\mu}
$$

where $A_{1}$ is the slip coefficient and $\sigma$ is the momentum accommodation coefficient (equal to one for perfectly diffuse molecular deflection, and zero for purely specular deflection). Kinetic theory and molecular simulations indicate that Maxwell's slip coefficient $\left(A_{1}=1\right)$ significantly overestimates the amount of actual velocity slip. Consequently, there is a large amount of fictitious slip incorporated within Maxwell's formulation, and this fortuitously improves its predictive capabilities when used with Navier-Stokes models. So here we use, instead, the actual (micro) slip, obtained by evaluating Equation (1) at the wall:

$$
A_{1}=\sqrt{\frac{2}{\pi}} \approx 0.8
$$

\section{Results and Discussion}

As a first test of our proposed wall-function approach, we use a simple centered finitedifference numerical scheme to solve the Navier-Stokes equations for monatomic gas flow in one-dimensional planar Couette and Poiseuille systems. Introducing the wallfunction into the calculation has negligible computational time penalty. Figures 2 and 3 show comparisons of solutions obtained using the Direct Simulation Monte Carlo 
(DSMC) method and the Navier-Stokes equations using both the conventional fictitious slip boundary conditions (with $A_{1}=1.146$ ) and the wall-function approach described above. In both cases the Knudsen number is defined as the ratio of the mean free path to half the channel height, $L$, and the shear stress profiles throughout the channel are identical for the three methods of simulation shown (i.e. the shear stress is unaffected by our wall-function).

Although the differences are fairly minor for these benchmark cases, results over a range of Mach numbers, $\mathrm{M}$, show that the wall-function approach produces better agreement with the DSMC data than conventional fictitious slip solutions do. Two further points to note are: a) both the wall-function and the fictitious slip approaches are based on the same kinetic theory, and corresponding assumptions, and are comparably easy to implement numerically; b) the Mach numbers considered here are reasonably high and slight viscous heating is exhibited in the DSMC data. To ensure a fair comparison with the isothermal continuum solutions, the mild variation of actual viscosity has been extracted from the DSMC data and accounted for within the Navier-Stokes calculations. In practice this only marginally affects the results.

We now investigate a non-planar case: low-Reynolds number (monatomic gas) flow past an unconfined sphere. Applying the wall-function approach to this case is no less, and no more, justifiable than the common practice of using fictitious boundary conditions in situations other than those for which they were originally derived. Figure 4 compares the total drag (normalized by the Stokes' drag — the limiting case as the Knudsen number tends to zero) of Milikan's experimentally-fitted curve for air ${ }^{9}$ with Navier-Stokes results using the Knudsen layer wall-function, and conventional 
slip-flow analysis (equivalent to a Navier-Stokes solution with slip coefficient $A_{1}=1$ ). Even though our wall-function is derived from a planar Knudsen layer solution, this method gives improved predictions of drag, particularly up to $\mathrm{Kn} \approx 0.1$ (which is the upper end of the Navier-Stokes slip flow regime). The gradient of the curve is also captured at low Knudsen numbers.

\section{Conclusions}

In this paper we have proposed a wall-function to scale the constitutive relations so that both the velocity slip and Knudsen layer structure can be captured within a continuum-fluid formulation for locally non-equilibrium gas flows. This wall-function is based on a simple Knudsen layer solution of the Boltzmann equation and improves the predictive capabilities of the Navier-Stokes equations for a variety of flow configurations of engineering interest. This method could be used as a simple and practical way of incorporating the Knudsen layer structure within conventional fluid dynamic simulations, although it is not comparable in either accuracy or complexity to the results from modern kinetic theory.

The technique could be used in conjunction with higher-order constitutive relations evaluated outside the Knudsen layer, and would then circumvent the need to provide additional boundary conditions for a unique solution. Future work includes implementing a more general Knudsen layer correction (such as that proposed by Sone ${ }^{3}$ ) using the wall-function approach, and investigating empirical Knudsen layer wall-functions for specific flow configurations at higher Knudsen numbers ${ }^{10}$. 


\section{Acknowledgments}

DAL and JMR are grateful to the UK's Leverhulme Trust (Research Project Grant No. F/07040/G) for supporting this research financially, and to Dr David Emerson and Dr Robert Barber of Daresbury Laboratory, UK, for very helpful discussions. MAG performed his work at Sandia National Laboratories. Sandia is a multiprogram laboratory operated by Sandia Corporation, a Lockheed Martin Company, for the United States Department of Energy's National Nuclear Security Administration under contract DE-AC04-94AL85000. 


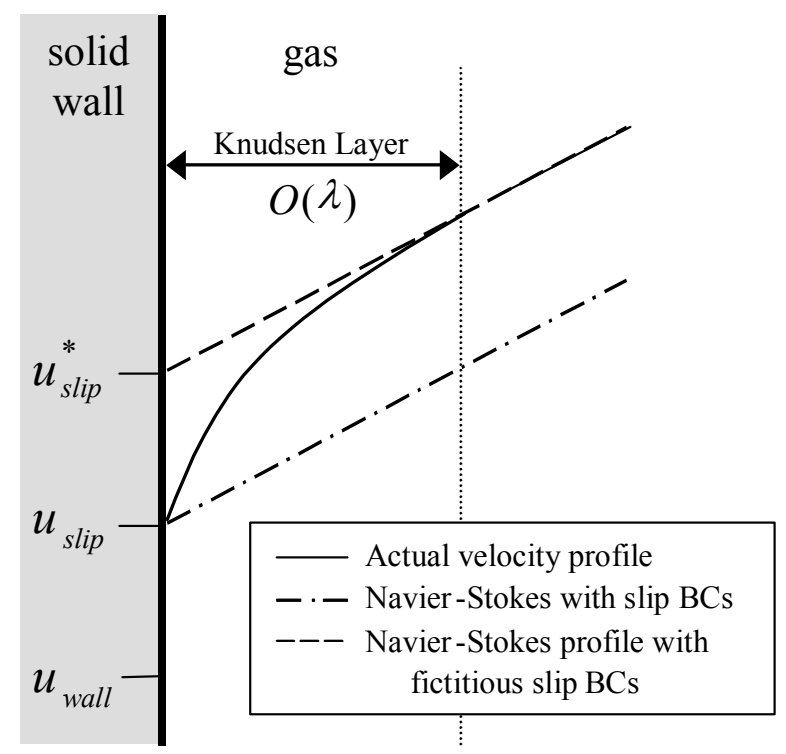

FIGURE 1. Schematic of the velocity structure of the Knudsen layer near a wall in a shear flow, with a comparison of the two types of slip boundary condition. 

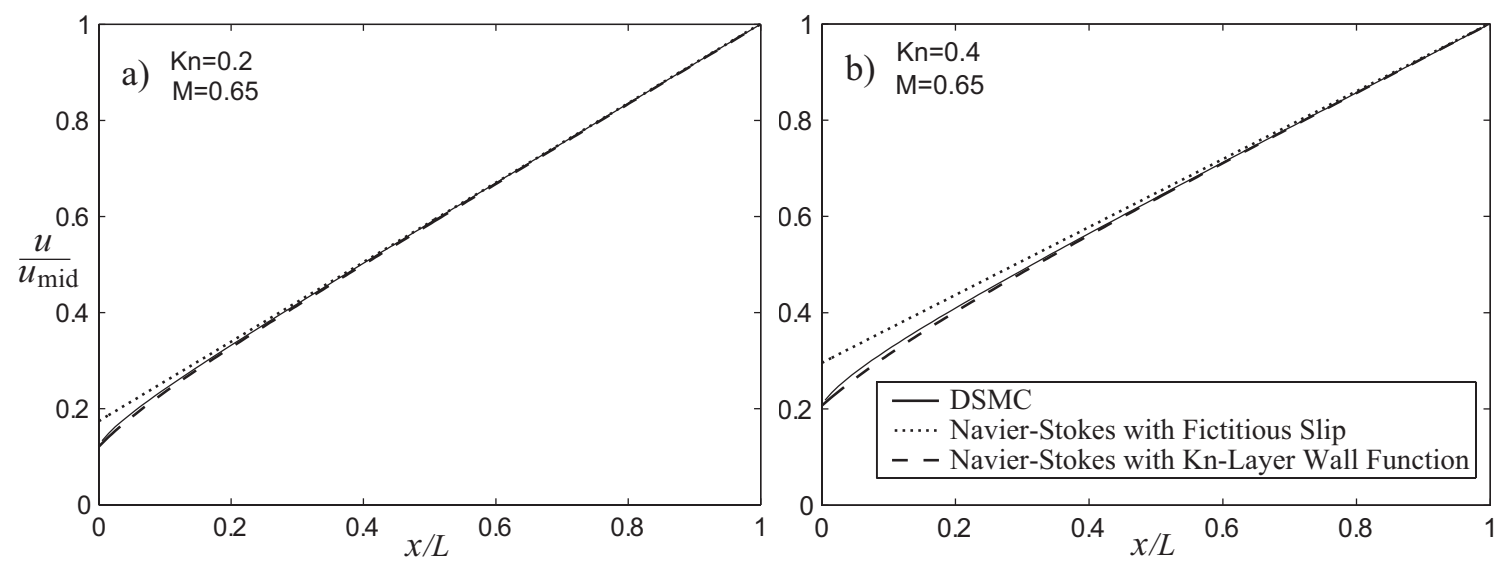

FIGURE 2. Normalised velocity profiles in rarefied Couette flow. DSMC results (一), Navier-Stokes solution with fictitious slip (․), Navier-Stokes solution with the Knudsen layer wall-function (- $)$. The wall at $x / L=0$ is stationary and that at $x / L=2$ is moving at Mach 0.65 with Knudsen numbers (a) $K n=0.2$ and (b) $K n=0.4$. 

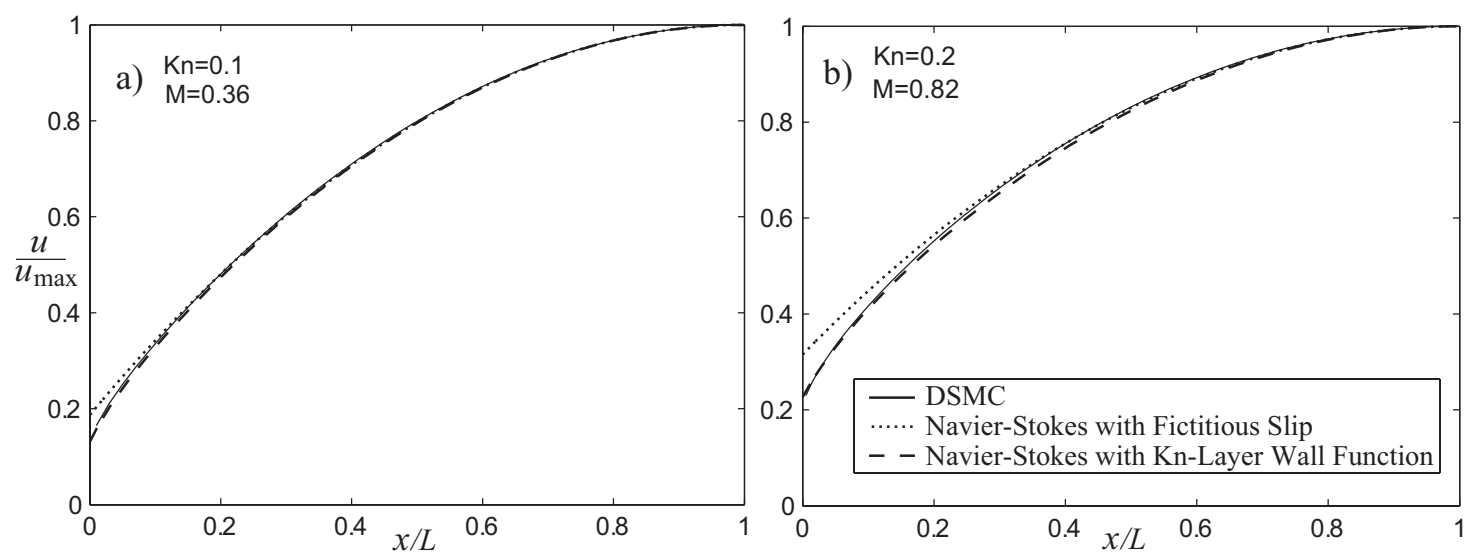

FIGURE 3. Normalized velocity profiles in rarefied Poiseuille flow. DSMC results (-), Navier-Stokes solution with fictitious slip (․), Navier-Stokes solution with the Knudsen layer wall-function (- ): (a) $\mathrm{Kn}=0.1, \mathrm{M}=0.36$; (b) $\mathrm{Kn}=0.2$, $\mathbf{M}=\mathbf{0 . 8 2}$. 


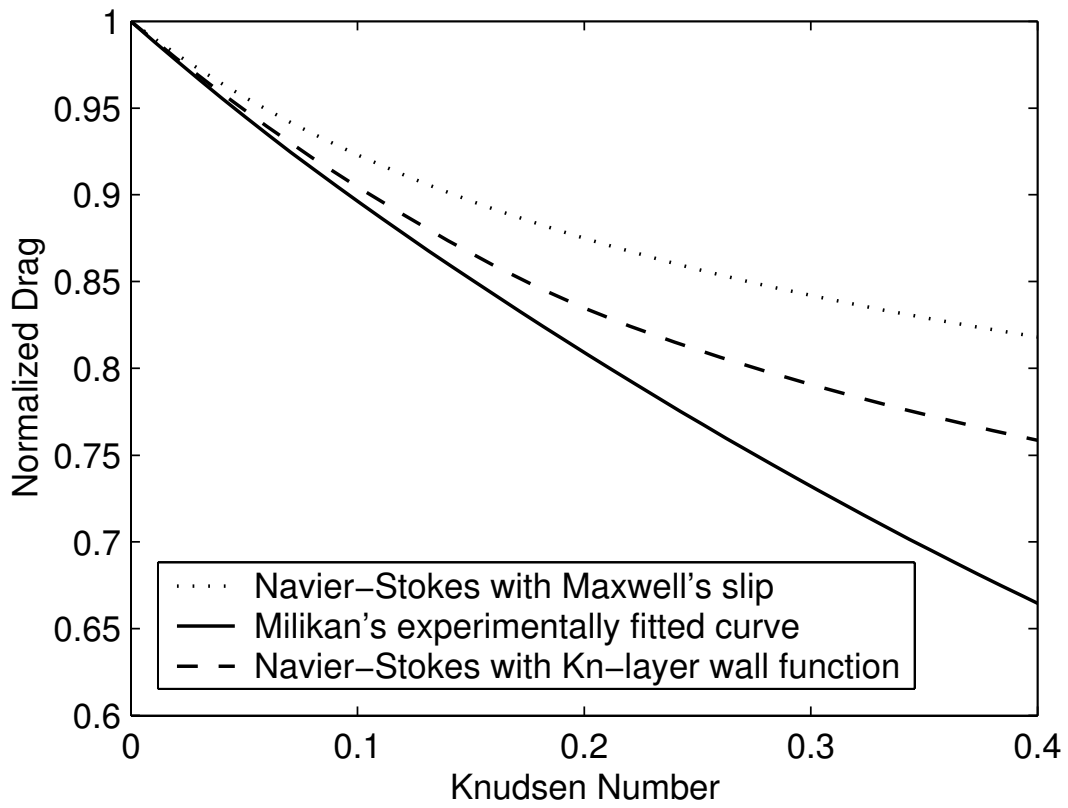

FIGURE 4. Normalized drag of an unconfined sphere over a range of Knudsen numbers. Experimentally-fitted curve ${ }^{9}(-)$, Navier-Stokes solution with Maxwell's slip (**), Navier-Stokes solution with the Knudsen layer wall-function $(--)$. The characteristic length used to define the Knudsen number is the sphere radius. 


\section{References}

${ }^{1}$ Kogan, M. N., Rarefied Gas Dynamics, Plenum Press, New York, 1969, p.367-399.

${ }^{2}$ Cercignani, C., Mathematical Methods in Kinetic Theory, Plenum Press, New York, 1990, p.232-243.

${ }^{3}$ Sone, Y., Kinetic Theory and Fluid Dynamics, Birkhauser, Boston, 2002

${ }^{4}$ Hadjiconstantinou, N. G., "Comment on Cercignani's second order slip coefficient" Phys. Fluids, Vol. 15, 2003, p. 2352-2354.

${ }^{5}$ Karniadakis, G. E., and Beskok, A., Microflows: Fundamentals and Simulation, Springer-Verlag, New York, 2001

${ }^{6}$ Lockerby, D. A., Reese, J. M., Barber, R. W., and Emerson, D. R., "Velocity boundary condition at solid walls in rarefied gas calculations" Phys. Rev. E Vol. 70, 2004, art. no. 017303.

${ }^{7}$ Cassell, J. S. and Williams, M. M. R., "An exact solution of the temperature slip problem in rarefied gases" Transport Theory Stat. Phys. Vol. 2(1), 1972, p.81-90.

${ }^{8}$ Maxwell, J. C., "On stresses in rarefied gases arising from inequalities of temperature" Phil. Trans. Roy. Soc. Vol. 170, 1879, p.231-256.

9 Allen, M. D., and Raabe, O. G., "Re-evaluation of Millikan's oil drop data for small particles in air" J. Aerosol Sci. Vol. 13, 1982, p.537-547.

${ }^{10}$ Lockerby, D. A., Reese, J. M., and Gallis, M. A., “A wall function approach to incorporating Knudsen-layer effects into gas micro flow simulations" Proceedings of the 24th International Symposium on Rarefied Gas Dynamics, Bari, Italy, 2004. 